\title{
Combined Treatment of Photodynamic Therapy and Bevacizumab for Choroidal Neovascularization Secondary to Age-Related Macular Degeneration
}

\author{
Hyun Woong Kim ${ }^{1}$, Jung Lim Kim ${ }^{1}$, Mi Hyun Lee ${ }^{2}$, Hyung Gon Yoo ${ }^{3}$, In Young Chung ${ }^{4}$, Ji Eun Lee \\ ${ }^{I}$ Department of Ophthalmology, Inje University College of Medicine, Busan, Korea \\ ${ }^{2}$ Department of Ophthalmology, Pusan National University School of Medicine, Yangsan, Korea \\ ${ }^{3}$ Department of Ophthalmology, Seoul National University College of Medicine, Seoul, Korea \\ ${ }^{4}$ Department of Ophthalmology, Gyeongsang National University School of Medicine, Jinju, Korea
}

\begin{abstract}
Purpose: To evaluate the outcome of a combined photodynamic therapy and intravitreal injection of bevacizumab in choroidal neovascularization secondary to age-related macular degeneration.

Methods: Photodynamic therapy (PDT) was administered to 28 eyes followed by 3 consecutive bevacizumab injections. Patients were followed-up for more than 12 months. At baseline, 1, 3, 6, and 12 months post PDT, visual acuity (VA) and central macular thickness were measured using optical coherence tomography.

Results: The mean VA was significantly improved from logarithm of the minimal angle of resolution 0.86 at baseline to 0.69 at 1 month $(p=0.011), 0.63$ at 3 months $(p=0.003), 0.64$ at 6 months $(p=0.004)$ and 0.60 at 12 months $(p<0.001)$. Central macular thickness decreased significantly from $328.3 \mu \mathrm{m}$ at baseline to $230.0 \mu \mathrm{m}$ at 6 months and $229.9 \mu \mathrm{m}$ at 1 year $(p<0.001)$. Reinjection mean number was 0.4 for 6 months and 0.8 for 12 months. By 1 year, retreatment was performed in 10 eyes (36\%).

Conclusions: PDT combined with three consecutive intraviteal bevacizumab injections was effective in improving $\mathrm{VA}$ and reducing central macular thickness.
\end{abstract}

Key Words: Bevacizumab, Choroidal neovascularization, Macular degeneration, Photochemotherapy, Verteporfin

Photodynamic therapy (PDT) with verteporfin (Visudyne; Novartis Pharma AG, Basel, Switzerland) has been shown to reduce the risk of moderate and severe vision loss in patients with choroidal neovascularization $(\mathrm{CNV})$ secondary to age-related macular degeneration (AMD). However, PDT rarely achieved visual improvement [1-3]. Choroidal hypoperfusion has been reported as a common side-effect after PDT [4]. Choroidal ischemia and its associated inflammation induce production of vascular endothelial growth factor (VEGF), which may lead to recurrence or new incidence of $\mathrm{CNV}[5,6]$. To manage recurrence, it is necessary to repeat PDT, which may result in choroid or retina atrophy and re-

Received: August 13, 2010 Accepted: November 12, 2010

Corresponding Author: Ji Eun Lee, MD, PhD. Department of Ophthalmology, Pusan National University Hospital, \#1-10 Ami-dong, Seo-gu, Busan 602-739, Korea. Tel: 82-51-505-0945, Fax: 82-51-242-7341, E-mail: jlee@pusan.ac.kr duce visual function [7]

Bevacizumab, a recombinant full-length humanized monoclonal antibody that binds all VEGF isoforms, was originally approved for treatment of metastatic carcinoma of the colon and rectum; however, it has been used as an off-label intravitreal treatment for CNV $[8,9]$. Several uncontrolled studies and case series have shown anatomical improvement and moderate vision gain when bevacizumab was used as a monotherapy [8-12]. However, repeated intravitreal injections are subject to the risk of complications, discomfort for the patient, and increased health care expense [13-16].

As CNV is a multi-factorial disease process, it was hypothesized that a combination therapy of different mechanisms of action would have additive or complementary treatment effects, and possibly result in the improvement of functional outcome as well as reduce the need for frequent retreatment [17]. Combination therapy has many options regarding sequence, interval, and number of each treatment given. Based on the report that the choroidal hypofluor- 
escence was found in indocyanine green (ICG) angiography for 3 months after PDT [4] and that the main functional improvement was achieved after 3 consecutive ranibizumab injections $[14,15,18,19]$, we conducted combined PDT and 3 consecutive intravitreal bevacizumab injections as treatment for patients with CNV secondary to AMD. This study was conducted to determine if this combination could achieve vision improvement and reduce the number and frequency of retreatment.

\section{Materials and Methods}

We retrospectively reviewed consecutive cases of exudative AMD that were treated with combined therapy of full-fluence PDT and 3 consecutive intravitreal injections of $1.25 \mathrm{mg}$ bevacizumab (Avastin; Genentech, South San Francisco, CA, USA) that were followed up for more than 1 year. All institutional review boards approved the off-label usage of bevacizumab and all patients signed an informed consent.

All patients were diagnosed with $\mathrm{CNV}$ due to wet AMD. The CNV diagnosis was confirmed by fundus examinations, fluorescein angiography (FA), and ICG angiography. Patients with a history of previous CNV treatment, or evidence of other disease that may cause CNV including myopic degeneration and angioid streaks, were excluded. Eyes with polypoidal choroidal vasculopathy were also excluded.

PDT was performed according to the Treatment of Age-Related Macular Degeneration with Photodynamic Therapy Study (TAP) regimen [20]. Greatest lesion diameter (GLD) was decided based on FA findings. Laser fluence and verteporfin (Visudyne, Novartis, Basel, Switzerland) doses were not reduced. Three consecutive intravitreal injections of $1.25 \mathrm{mg}$ bevacizumab were performed within 3 days, at 1 month, and 2 months post PDT.

Best corrected visual acuity (VA) measurement, fundus examination, and optical coherence tomography (OCT; Stratus OCT, Carl Zeiss Meditec Inc., Dublin, CA, USA) were performed at each visit. OCT scans were performed using the Fast macular thickness map protocol. Scans with signal strength of 6 or higher were used for analysis. If a radial scan contained a segmentation algorithm error in the central $1 \mathrm{~mm}$, it was excluded from map calculation. FA was performed to assess lesion activity when clinically indicated. Additional treatment was decided at the physician's discretion based on previous reports [1-3,21]. Retreatment of either PDT or intravitreal bevacizumab were performed in the following cases: when a VA loss of at least 1 line with OCT evidence of fluid in the macula occurred, when an increase in central subfield macular thickness of at least $100 \mu \mathrm{m}$ was present, when a new area of classic CNV with macular hemorrhage in FA developed, or when persistent subretinal or intraretinal fluid on OCT occurred.

VA was measured using a standard Snellen chart and was converted to logarithm of the minimum angle of resolution
( $\log$ MAR) for statistical analysis. Improved or worsened VA was defined as a change of 0.3 or more in the logMAR score. The main outcome measures were changes in VA and central subfield macular thickness during the 12-month follow-up period. The occurrences of retreatment were also evaluated. Statistical analysis was performed with SPSS ver. 13.0 (SPSS Inc., Chicago, IL, USA).

\section{Results}

Twenty-eight eyes from 28 consecutive patients (18 men and 10 women) were included in this study. Baseline characteristics are summarized in Table 1. The mean patient age was 67.8 years and the patients were followed up for an average of 17.2 months (range, 12 to 33 months). The type of $\mathrm{CNV}$ was predominantly classic in 8 eyes, minimal classic in 4 eyes, and occult in 16 eyes. The mean GLD was $2,840 \mu \mathrm{m}$ ( $\pm 1,033$; range, 900 to 5,220 ).

VA ( $\log$ MAR) was 0.86 at baseline, 0.69 at 1 month $(p=$ $0.011), 0.63$ at 3 months ( $p=0.003), 0.64$ at 6 months ( $p=$ $0.004)$ and 0.60 at 1 year $(p<0.001)$ (Fig. 1A). The difference between baseline and every other visit was statistically significant (Wilcoxon signed rank sum test). VA improved by 3 lines or more in 13 eyes (46.4\%) at 6 months and in 12 eyes $(42.9 \%)$ at 1 year (Table 2$)$. VA decreased by 3 lines or more in 2 eyes $(7.1 \%)$ at 6 months and 1 eye $(3.6 \%)$ at 1 year. GLD significantly correlated with VA at 1 year $(p<0.001)$, but not with baseline acuity or acuity change (Spearman's ranked correlation). The mean central subfield macular thickness decreased significantly from $328.3 \mu \mathrm{m}$ at baseline to $230.0 \mu \mathrm{m}$ at 6 months and $229.9 \mu \mathrm{m}$ at 1 year $(p<0.001)$ (Fig. 1B).

In the 8 patients with predominantly classic lesions, VA changed from a $\log$ MAR of 1.15 at baseline to 0.73 at 6 months and 0.71 at 1 year. VA did not change in 4 eyes $(50 \%)$ and improved in 4 eyes (50\%). In the 16 eyes with occult CNV, VA was 0.79 at baseline, 0.69 at 6 months, and 0.64 at

Table 1. Baseline demographic and lesion characteristics for patients with more than 6 months follow up

\begin{tabular}{lc}
\hline \multicolumn{1}{c}{ Characteristic } \\
\hline No. of patients & 28 \\
Age $(\mathrm{yr})$ & $67.8(55-82)$ \\
Gender $(\%)$ & \\
$\quad$ Male & $18(64)$ \\
$\quad$ Female & $10(36)$ \\
Visual acuity (Snellen) & $0.2(0.02-0.8)$ \\
Visual acuity (logMAR) & $0.86( \pm 0.49)$ \\
Central subfield retinal thickness $(\mu \mathrm{m})$ & $328.3(180-570)$ \\
Lesion type $(\%)$ & \\
$\quad$ Predominant classic & $8(29)$ \\
$\quad$ Minimal classic & $4(14)$ \\
$\quad$ Occult without classic & $16(57)$ \\
$\quad$ Greatest linear diameter $(\mu \mathrm{m})$ & $2,840(900-5,220)$ \\
\hline
\end{tabular}

$\log \mathrm{MAR}=$ logarithm of the minimum angle of resolution. 

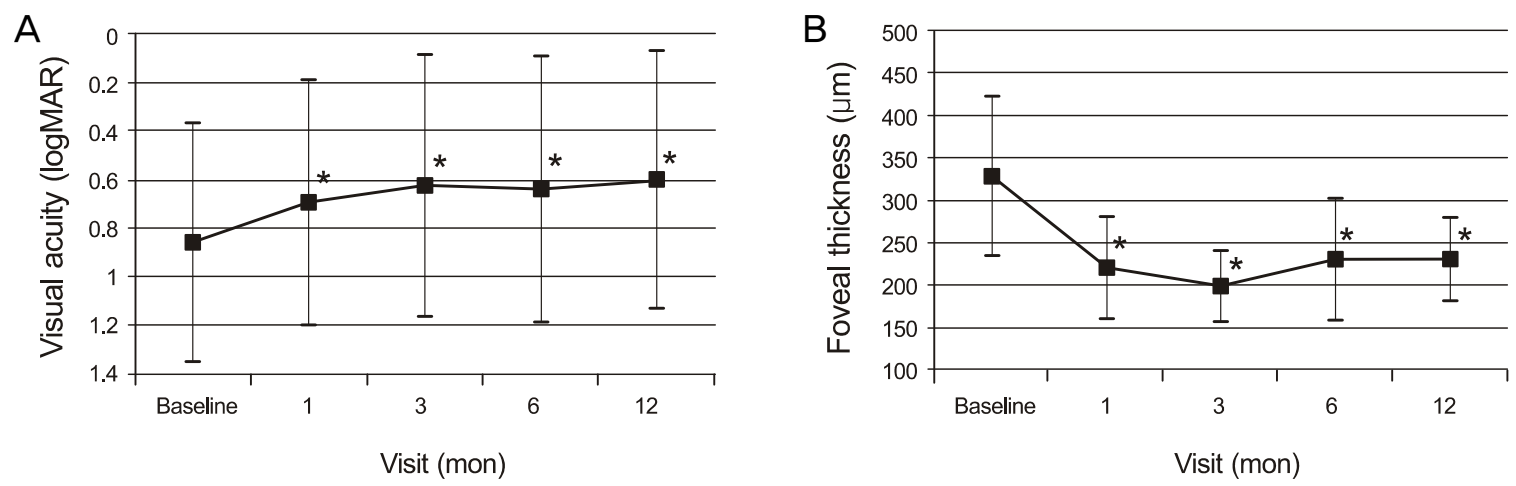

Fig. 1. Mean visual acuity (A) and mean central retinal thickness on optical coherence tomography (B) before and at 12 months after combined treatment of photodynamic therapy and 3 consecutive monthly bevacizumab injections. $\log$ MAR $=$ logarithm of the minimal angle of resolution. ${ }^{*} p<0.05$, Wilcoxon signed rank sum test.

Table 2. Visual acuity changes after combined treatment of photodynamic therapy and 3 consecutive monthly bevacizumab injections for choroidal neovascularization complicated with age-related macular degeneration

\begin{tabular}{|c|c|c|c|c|c|}
\hline & Baseline & $1 \mathrm{mon}$ & 3 mon & $6 \mathrm{mon}$ & $12 \mathrm{mon}$ \\
\hline Mean visual acuity (logMAR) & 0.86 & 0.71 & 0.63 & 0.64 & 0.60 \\
\hline \multicolumn{6}{|l|}{ Visual acuity distribution (eyes) } \\
\hline$<20 / 200$ & 7 & 5 & 6 & 6 & 6 \\
\hline $20 / 200-20 / 40$ & 18 & 16 & 8 & 9 & 9 \\
\hline$\geq 20 / 40$ & 3 & 7 & 14 & 13 & 13 \\
\hline \multicolumn{6}{|l|}{ Visual acuity changes (eyes) } \\
\hline$\geq 3$ line decrease & & 3 & 2 & 2 & 1 \\
\hline Change of 2 lines or less & & 16 & 14 & 13 & 15 \\
\hline$\geq 3$ line improvement & & 9 & 12 & 13 & 12 \\
\hline
\end{tabular}

$\log M A R=$ logarithm of the minimum angle of resolution.

A

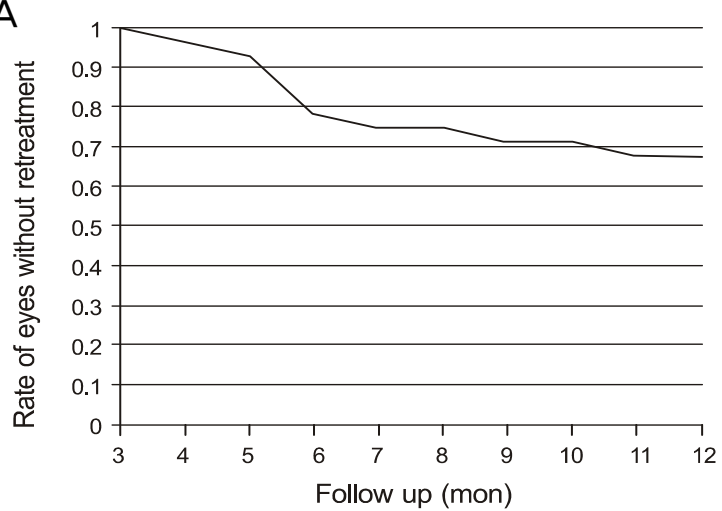

B

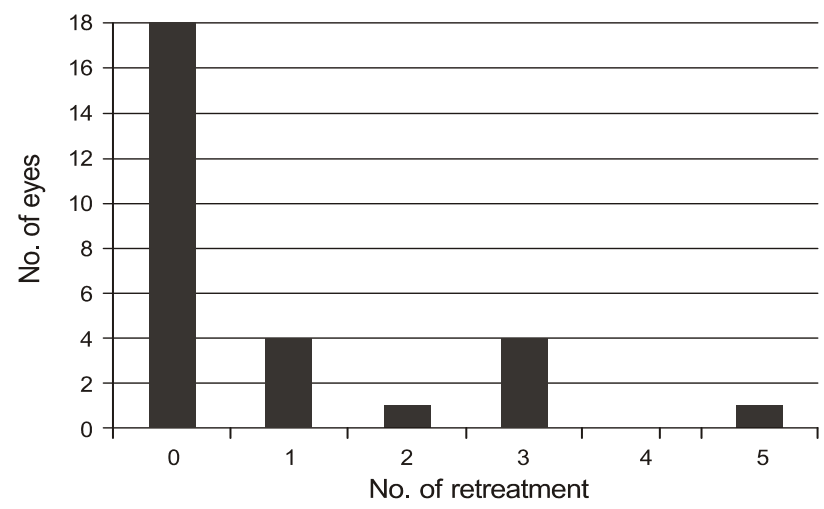

Fig. 2. (A) Kaplan-Meyer analysis represents the number of eyes that did not require additional treatment after combined photodynamic therapy and 3 consecutive monthly bevacizumab injections. (B) Numbers of retreatment from 3 to 12 months.

1 year. VA improved in 5 eyes (31\%) and decreased in 1 eye $(3 \%)$. The difference in visual improvement among lesion type was not statistically significant (Kruskal-Wallis test, $p>0.05$ ).

The mean number for reinjection was 0.4 at 6 months and 0.8 at 12 months. At 6 months, 10 injections of bevacizumab and 2 PDT were performed in 7 eyes as additional treatments (23.3\%) (Figs. 2 and 3). Between months 7 and 12, 6 eyes $(20.0 \%)$ required 13 injections and 1 PDT (Fig.
2). Overall, retreatment was required in 10 eyes (33.3\%), and 18 eyes $(56.7 \%)$ did not require additional anti-VEGF injections or PDT other than the initial combined treatment for 12 months of follow up (Figs. 2 and 4). There were no intravitreal injection-related complications such as endophthalmitis, glaucoma, retinal detachment, uveitis, or other non-ocular complications. 

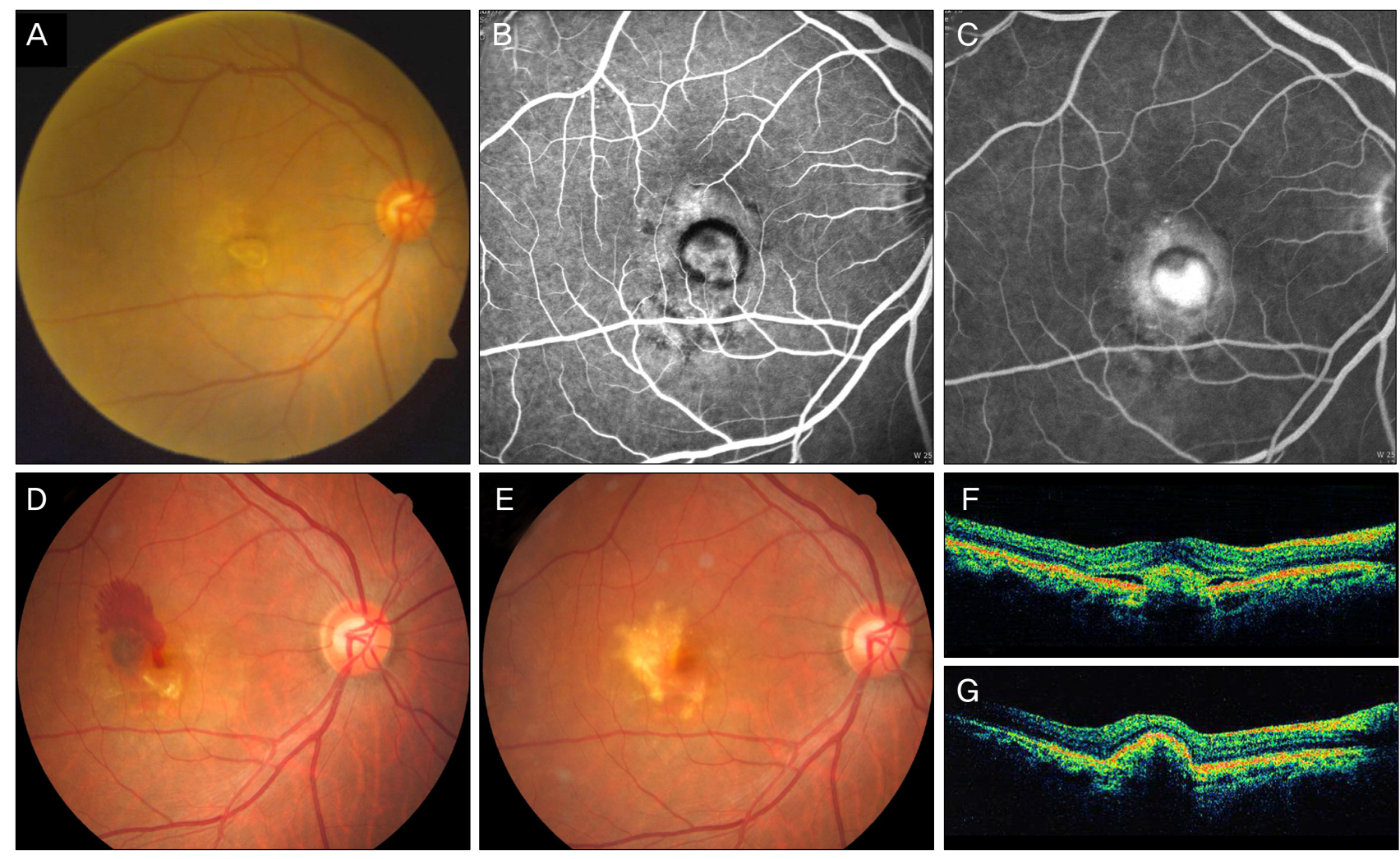

Fig. 3. (A) A 59-year old woman with a visual acuity (VA) score of $0.02 \mathrm{had}$ classic choroidal neovascularization revealed in early (B) and late phase (C) fluorescein angiography. At 5 months after combined photodynamic therapy and 3 monthly bevacizumab injections a new subretinal hemorrhage developed. (E) At 1 year, VA improved to 0.6 after two additional bevacizumab injections. (F) Optical coherence tomography shows choroidal neovascularization and associated subretinal fluid before treatment. (G) Subretinal fluid resolved completely with combined treatment and two injections for 1 year.

\section{Discussion}

Although PDT monotherapy had better functional results than the sham treatment, patients had an average loss of 2.2 and 3.1 lines of vision in the previous TAP and Verteporfin in Photodynamic Therapy studies, respectively $[1,20]$. In the verteporfin treatment group of the anti-VEGF antibody for the treatment of predominantly classic choroidal neovascularisation in AMD (ANCHOR) study, the mean VA decrease was 9.5 letters [15]. PDT with verteporfin is relatively selective for $\mathrm{CNV}$; however, it may cause ischemia of the normal choroidal vessels [4]. Following reactive hypoxia, proinflammatory cytokine and VEGF can promote recurrence of CNV. The repeated PDT insults may result in RPE and choroidal atrophy. To improve the functional results of PDT, it is important to control inflammation related to PDT. This is supported by several reports that have shown a beneficial effect on vision from PDT combined with triamcinolone acetonide intravitreal administration [22-24].

Anti-VEGF therapy with ranibizumab has shown better visual outcomes in prospective multicenter studies. The MARINA study, in which patients received intravitreal injections of ranibizumab monthly for 2 years, showed a mean improvement in VA of 6.5 and 7.2 ETDRS letters for the
$0.3 \mathrm{mg}$ and $0.5 \mathrm{mg}$ ranibizumab doses, respectively [14]. However, these results were based on frequent injections at fixed intervals every month, which increases the risk of endophthalmitis or retinal detachment complications as well as increasing economic burden. Therefore, many studies have been conducted to decrease the number of intravitreal injections without deterioration of the functional outcomes of anti-VEGF treatment.

The Prospective OCT Imaging of Patients with Neovascular AMD Treated with Intraocular Ranibizumab (PrONTO) study involved 3 scheduled ranibizumab injections and then as required based on clinical findings. The mean improvement in VA was 9.3 Early Treatment Diabetic Retinopathy Study (ETDRS) letters with an average of 5.6 injections over 12 months, and 11.1 letters with 9.9 injections over 24 months $[25,26]$. Improvement in the $\log M A R$ score $(0.26)$ in this study is comparable to the PrONTO study.

Inhibition of VEGF suppresses the development and maintenance of neovascularization but is less efficient on established neovascularization, which is assumed to be the cause of the high recurrence rate after anti-VEGF treatment $[17,18]$. In contrast, PDT with verteporfin treatment was shown to induce occlusion of mature new vessels, which are not inhibited by anti-VEGF agents [27]. Therefore, the com- 

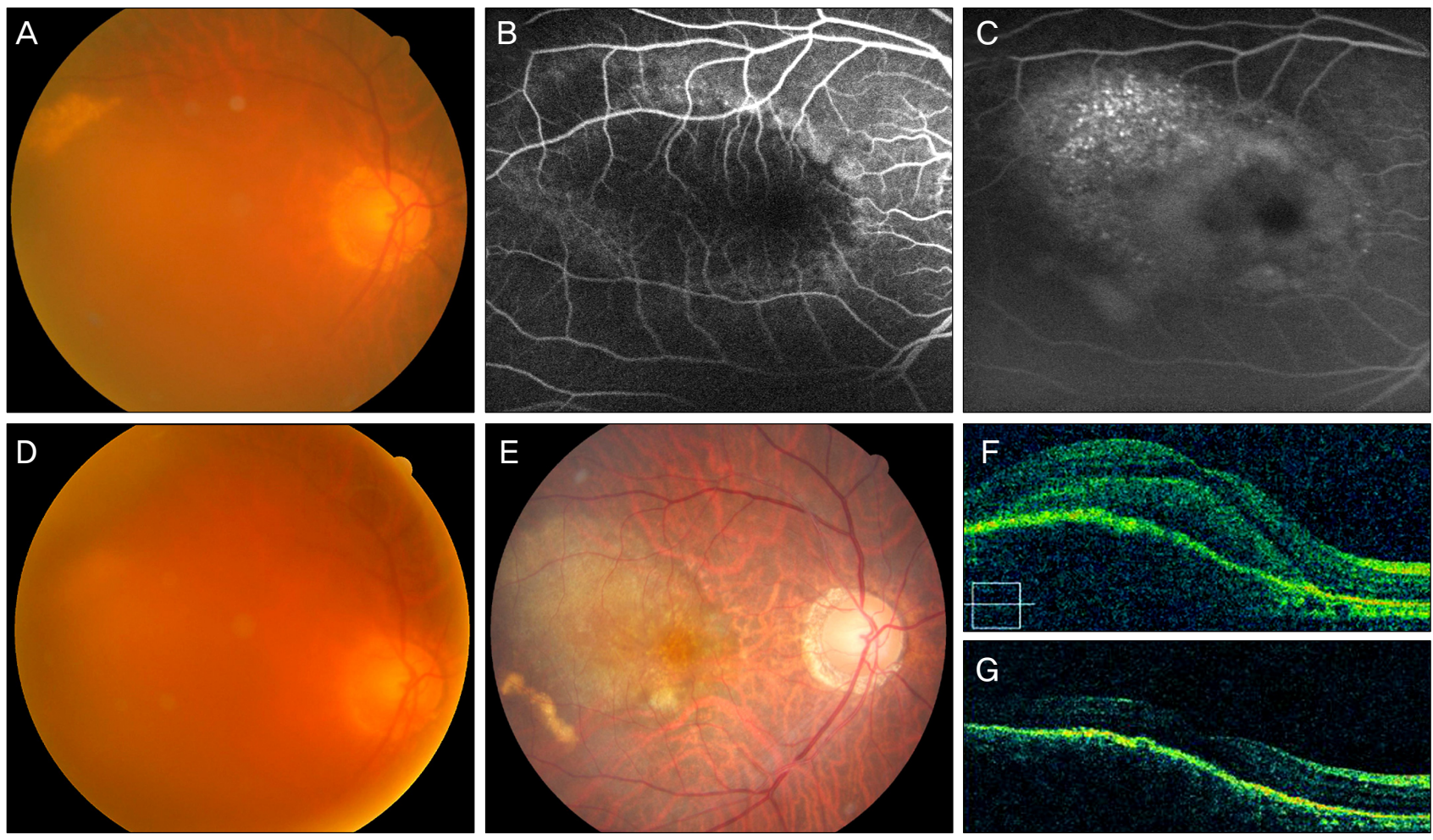

Fig. 4. (A) A 76-year old man presented with age-related macular degeneration in the right eye. Posterior subcapsular opacity blurs the posterior pole. The visual acuity (VA) score was 0.2 . Fluorescein angiography of the early (B) and late (C) phase shows occult choroidal neovascularization with active leakage. (D) After combined treatment of photodynamic therapy and 3 monthly bevacizumab injections, VA increased to 0.6. (E) At 1 year, he underwent cataract surgery. VA remained at 0.6 without retreatment. Optical coherence tomography at presentation (F) shows subretinal fluid, which resolved 3 months after the combined treatment $(\mathrm{G})$.

bination of PDT with anti-angiogenic agents is expected to have some additive benefits related to VA improvement and reduction of treatment rates.

In the first prospective multicenter trial of combination PDT and ranibizumab treatment (the ranibizumab combined with verteporfin photodynamic therapy in neovascular age related macular degeneration [FOCUS] study), PDT was performed at baseline and then as required, and monthly ranibizumab injections were administered [28]. Although the combined treatment showed better visual improvements than PDT alone, the injection rate was not reduced.

Several studies have reported the results of PDT combined with a single intravitreal injection of anti-VEGF. Smith et al. suggested that the mean improvement in VA was 1.57 lines by a single intravitreal injection with bevacizumab followed by PDT at the final 12 month follow up [29]. Mean numbers of retreatment per eye were 0.57 during the 12 month follow up. Navea et al treated 63 patients using bevacizumab intravitreal injection followed by PDT for 12 months [16]. The mean numbers for additional PDT and additive bevacizumab injections per patient were 0.46 and 1.0, respectively. No additional treatment for recurrence was required in $46 \%$ of the cases. Although the results of the above studies cannot be compared directly with the MARINA and ANCHOR trials, the combined therapy seems promising as a method to reduce

\section{the need for retreatment.}

In the current study, eyes with exudative AMD were treated using 3 consecutive intravitreal bevacizumab injections following PDT. Our protocol was based on the following aspects. First, according to the MARINA and ANCHOR studies, VA was most improved after the first 3 monthly intravitreal ranibizumab injections $[14,15,18,19]$. Second, choroidal hypofluorescence may be noted in ICG angiography for 3 months after PDT. Using this protocol, we expected to achieve more functional improvement and less retreatment.

Compared to the results of studies using PDT combined with a single injection, our protocol resulted in a reduced number of injections. However, 3 consecutive monthly injections did not eliminate recurrence, and considering the number of the basic treatments, more injections were performed in the current study.

Regarding the functional results, VA was maintained or improved in $96.4 \%$ of eyes at 1 year. However, the number of eyes with poor VA (less than 20 / 200) did not change during follow up (Table 2). Final VA significantly correlated with GLD, which represents lesion size. These results indicate that it would be difficult to restore VA with the combined treatment in cases where CNV had already enlarged and visual function had severely deteriorated.

In spite of choroidal hypofluorescence in ICG after PDT, 
clinically significant choroidal ischemia is not uncommon $[4,6,30]$. As VEGF may have a physiologic role in preventing significant infarction and recovering normal choroidal perfusion as well as inducing pathologic $\mathrm{CNV}$, reduced fluence could result in better outcomes in combination treatment with PDT and anti-VEGF. In the phase 2 Reduced Fluence Visudyne PDT Combined with Anti-VEGF and Dexamethasone in Combination for AMD Lesions (RADICAL) study, the triple therapy showed superior VA and decreased treatment frequency compared with anti-VEGF monotherapy [31].

In conclusion, the combination treatment of PDT and 3 consecutive anti-VEGF injections resulted in improved visual function and macular anatomy for 1 year. The correlation of GLD with VA at 1 year suggests that early diagnosis and intervention is an important factor for visual prognosis. This study was limited by its retrospective design and small sample size without controls. Further studies on various treatment combinations with reduced fluence PDT and antiVEGF are necessary. Moreover, further studies regarding retreatment criteria and methods with larger controlled trials are required to design the optimal treatment guide for neovascular AMD.

\section{Conflict of Interest}

No potential conflict of interest relevant to this article was reported.

\section{Acknowledgements}

This work was supported by the 2009 Inje University research grant.

\section{References}

1. Verteporfin in Photodynamic Therapy Study Group. Verteporfin therapy of subfoveal choroidal neovascularization in age-related macular degeneration: two-year results of a randomized clinical trial including lesions with occult with no classic choroidal neovascularization--verteporfin in photodynamic therapy report 2. Am J Ophthalmol 2001;131:541-60.

2. Azab M, Boyer DS, Bressler NM, et al. Verteporfin therapy of subfoveal minimally classic choroidal neovascularization in age-related macular degeneration: 2-year results of a randomized clinical trial. Arch Ophthalmol 2005;123:448-57.

3. Bressler NM; Treatment of Age-Related Macular Degeneration with Photodynamic Therapy (TAP) Study Group. Photodynamic therapy of subfoveal choroidal neovascularization in age-related macular degeneration with verteporfin: two-year results of 2 randomized clinical trials-tap report 2. Arch Ophthalmol 2001;119:198-207.

4. Schmidt-Erfurth U, Michels S, Barbazetto I, Laqua H. Photodynamic effects on choroidal neovascularization and physiological choroid. Invest Ophthalmol Vis Sci 2002;43:830-41.

5. Matsuoka M, Ogata N, Otsuji T, et al. Expression of pigment epithelium derived factor and vascular endothelial growth factor in choroidal neovascular membranes and polypoidal choroidal vasculopathy. Br J Ophthalmol 2004;88:809-15.

6. Schmidt-Erfurth U, Schlotzer-Schrehard U, Cursiefen C, et al.
Influence of photodynamic therapy on expression of vascular endothelial growth factor (VEGF), VEGF receptor 3, and pigment epithelium-derived factor. Invest Ophthalmol Vis Sci 2003;44:4473-80.

7. Schmidt-Erfurth U, Miller JW, Sickenberg M, et al. Photodynamic therapy with verteporfin for choroidal neovascularization caused by age-related macular degeneration: results of retreatments in a phase 1 and 2 study. Arch Ophthalmol 1999;117: 1177-87.

8. Avery RL, Pieramici DJ, Rabena MD, et al. Intravitreal bevacizumab (Avastin) for neovascular age-related macular degeneration. Ophthalmology 2006;113:363-372.e5.

9. Spaide RF, Laud K, Fine HF, et al. Intravitreal bevacizumab treatment of choroidal neovascularization secondary to age-related macular degeneration. Retina 2006;26:383-90.

10. Bashshur ZF, Bazarbachi A, Schakal A, et al. Intravitreal bevacizumab for the management of choroidal neovascularization in age-related macular degeneration. Am J Ophthalmol 2006; 142:1-9.

11. Costa RA, Jorge R, Calucci D, et al. Intravitreal bevacizumab for choroidal neovascularization caused by AMD (IBeNA Study): results of a phase 1 dose-escalation study. Invest Ophthalmol Vis Sci 2006;47:4569-78.

12. Rich RM, Rosenfeld PJ, Puliafito CA, et al. Short-term safety and efficacy of intravitreal bevacizumab (Avastin) for neovascular age-related macular degeneration. Retina 2006;26: 495-511.

13. Gragoudas ES, Adamis AP, Cunningham ET Jr, et al. Pegaptanib for neovascular age-related macular degeneration. $N$ Engl $J$ Med 2004;351:2805-16.

14. Rosenfeld PJ, Brown DM, Heier JS, et al. Ranibizumab for neovascular age-related macular degeneration. $N$ Engl J Med 2006;355:1419-31.

15. Brown DM, Kaiser PK, Michels M, et al. Ranibizumab versus verteporfin for neovascular age-related macular degeneration. N Engl J Med 2006;355:1432-44.

16. Navea A, Mataix J, Desco MC, et al. One-year follow-up of combined customized therapy. Photodynamic therapy and bevacizumab for exudative age-related macular degeneration. Retina 2009;29:13-9.

17. Kaiser PK. Verteporfin photodynamic therapy and anti-angiogenic drugs: potential for combination therapy in exudative age-related macular degeneration. Curr Med Res Opin 2007; 23:477-87.

18. Kaiser PK, Blodi BA, Shapiro H, et al. Angiographic and optical coherence tomographic results of the MARINA study of ranibizumab in neovascular age-related macular degeneration. Ophthalmology 2007;114:1868-75.

19. Kaiser PK, Brown DM, Zhang K, et al. Ranibizumab for predominantly classic neovascular age-related macular degeneration: subgroup analysis of first-year ANCHOR results. Am J Ophthalmol 2007; 144:850-7.

20. Treatment of Age-related Macular Degeneration with Photodynamic Therapy (TAP) Study Group. Photodynamic therapy of subfoveal choroidal neovascularization in age-related macular degeneration with verteporfin: one-year results of 2 randomized clinical trials: TAP report. Arch Ophthalmol 1999;117:1329-45.

21. Lalwani GA, Rosenfeld PJ, Fung AE, et al. A variable-dosing regimen with intravitreal ranibizumab for neovascular age-related macular degeneration: year 2 of the PrONTO Study. Am J Ophthalmol 2009;148:43-58.e1.

22. Rechtman E, Danis RP, Pratt LM, Harris A. Intravitreal triamcinolone with photodynamic therapy for subfoveal choroidal neovascularisation in age related macular degeneration. $\mathrm{Br}$ J Ophthalmol 2004;88:344-7. 
23. Spaide RF, Sorenson J, Maranan L. Photodynamic therapy with verteporfin combined with intravitreal injection of triamcinolone acetonide for choroidal neovascularization. Ophthalmology 2005; 112:301-4.

24. Danis RP, Ciulla TA, Pratt LM, Anliker W. Intravitreal triamcinolone acetonide in exudative age-related macular degeneration. Retina 2000;20:244-50.

25. Spaide RF. Rationale for combination therapies for choroidal neovascularization. Am J Ophthalmol 2006;141:149-56.

26. Fung AE, Lalwani GA, Rosenfeld PJ, et al. An optical coherence tomography-guided, variable dosing regimen with intravitreal ranibizumab (Lucentis) for neovascular age-related macular degeneration. Am J Ophthalmol 2007;143:566-83.

27. Benjamin LE, Golijanin D, Itin A, et al. Selective ablation of immature blood vessels in established human tumors follows vascular endothelial growth factor withdrawal. J Clin Invest
1999;103:159-65.

28. Heier JS, Boyer DS, Ciulla TA, et al. Ranibizumab combined with verteporfin photodynamic therapy in neovascular age-related macular degeneration: year 1 results of the FOCUS Study. Arch Ophthalmol 2006;124:1532-42.

29. Smith BT, Dhalla MS, Shah GK, et al. Intravitreal injection of bevacizumab combined with verteporfin photodynamic therapy for choroidal neovascularization in age-related macular degeneration. Retina 2008;28:675-81.

30. Isola V, Pece A, Parodi MB. Choroidal ischemia after photodynamic therapy with verteporfin for choroidal neovascularization. Am J Ophthalmol 2006;142:680-3.

31. Augustin AJ, Puls S, Offermann I. Triple therapy for choroidal neovascularization due to age-related macular degeneration: verteporfin PDT, bevacizumab, and dexamethasone. Retina 2007;27:133-40. 